\title{
THE UNIVERSITY of EDINBURGH
}

\author{
Edinburgh Research Explorer
}

\section{Determination of the helicity of oriented photofragments}

\section{Citation for published version:}

Alexander, AJ 2005, 'Determination of the helicity of oriented photofragments', Journal of Chemical Physics, vol. 123, no. 19, 194312, pp. -. https://doi.org/10.1063/1.2122667

\section{Digital Object Identifier (DOI):}

$10.1063 / 1.2122667$

Link:

Link to publication record in Edinburgh Research Explorer

Document Version:

Publisher's PDF, also known as Version of record

Published In:

Journal of Chemical Physics

Publisher Rights Statement:

Copyright 2005 American Institute of Physics. This article may be downloaded for personal use only. Any other use requires prior permission of the author and the American Institute of Physics.

\section{General rights}

Copyright for the publications made accessible via the Edinburgh Research Explorer is retained by the author(s) and / or other copyright owners and it is a condition of accessing these publications that users recognise and abide by the legal requirements associated with these rights.

\section{Take down policy}

The University of Edinburgh has made every reasonable effort to ensure that Edinburgh Research Explorer content complies with UK legislation. If you believe that the public display of this file breaches copyright please contact openaccess@ed.ac.uk providing details, and we will remove access to the work immediately and investigate your claim. 


\section{AIP}

\section{Determination of the helicity of oriented photofragments}

Andrew J. Alexander

Citation: J. Chem. Phys. 123, 194312 (2005); doi: 10.1063/1.2122667

View online: http://dx.doi.org/10.1063/1.2122667

View Table of Contents: http://jcp.aip.org/resource/1/JCPSA6/v123/i19

Published by the AIP Publishing LLC.

\section{Additional information on J. Chem. Phys.}

Journal Homepage: http://jcp.aip.org/

Journal Information: http://jcp.aip.org/about/about_the_journal

Top downloads: http://jcp.aip.org/features/most_downloaded

Information for Authors: http://jcp.aip.org/authors

\section{ADVERTISEMENT}

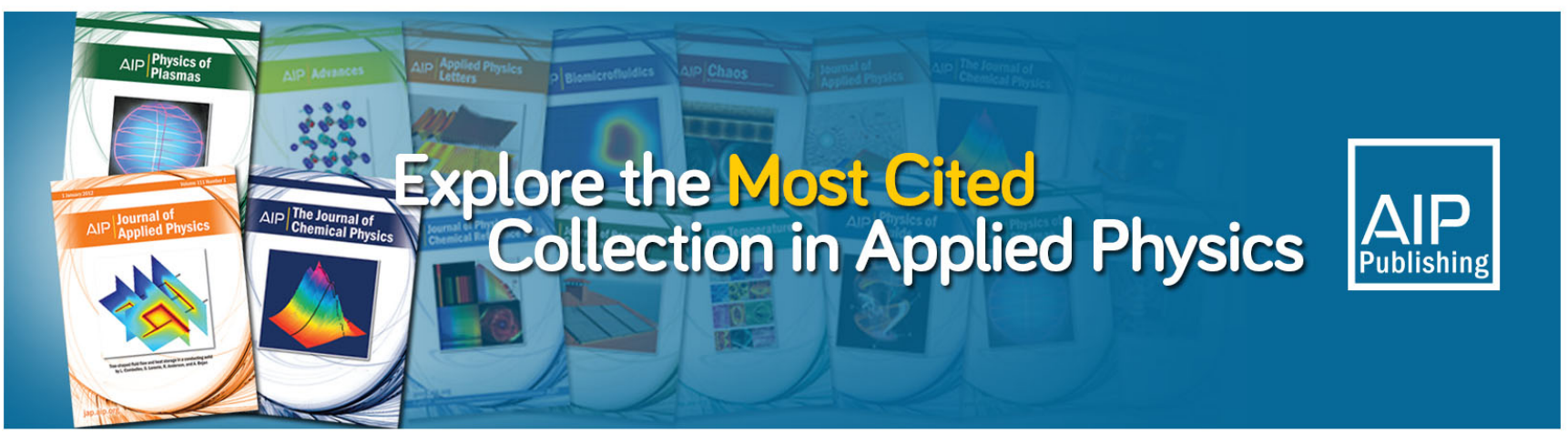




\title{
Determination of the helicity of oriented photofragments
}

\author{
Andrew J. Alexander ${ }^{a}$ \\ School of Chemistry, University of Edinburgh, West Mains Road, Edinburgh EH9 3JJ, United Kingdom
}

(Received 15 September 2005; accepted 23 September 2005; published online 17 November 2005)

\begin{abstract}
Equations to enable determination of the helicity (angular momentum orientation) of photofragments resulting from single-photon dissociation of an isotropic sample of molecules are presented. The symmetry of the photofragment distribution is illustrated by three-dimensional vector plots of the expectation values of projections of the fragment total angular momentum. Equations describing circular polarization of light in the spherical tensor basis are presented. Methods for the optical measurement of angular momentum orientation are discussed, including determination of the helicity of circularly polarized light by a quarter-wave plate or single Fresnel rhomb. (C) 2005 American Institute of Physics. [DOI: 10.1063/1.2122667]
\end{abstract}

\section{INTRODUCTION}

Dissociation of samples of molecules using polarized radiation can be used to further our understanding of the dynamics of fragmentation processes. By using polarized photolysis light, we create a frame of reference in the laboratory in which we can describe spatial features of the products, such as their velocities and angular momentum distributions. These reflect both the electronic states populated in the initial excitation step and the forces acting during the dissociation, and provide considerable insight into the mechanism of bond breakage. Of particular interest are spatial measurements of product angular momentum $J .^{1,2}$ For a molecule, this could mean the sense of rotation: the distinction between clockwise or counterclockwise is called orientation. Similarly, the angular momenta of the electrons in a fragment atom can be oriented. Put a different way, we could say that the populations of $+M_{J}$ sublevels are different from $-M_{J}$, resulting in an orientation along a quantization axis $z$. We may also speak of fragment alignment, which indicates a preferred plane of rotation with respect to $z$, irrespective of whether the motion is clockwise or counterclockwise, e.g., a frisbee or a cartwheel. ${ }^{3}$ Dissymmetry in the populations of sublevels $M_{J}$ is not the only cause of polarization; the phase differences between sublevels can produce polarization along axes perpendicular to the $z$ axis. The populations and phases give the density matrix $\rho_{M M^{\prime}}{ }^{4}$

Rather than specify all of the populations and phases in the density matrix, it is useful to define the (complex) polarization moments $A_{q}^{(k)}$ and $A_{q}^{(k)^{*}}=(-1)^{q} A_{q_{1,3}}^{(k)}$, where $k$ $=0, \ldots, 2 J$, with $q=-k, \ldots,+k$, and $A_{0}^{(0)} \equiv 1.1,3$ The odd $k$ moments describe orientation; even $k$ moments describe alignment. Each moment neatly distils a complicated collection of $M_{J}$ populations and phases into a single value that can be used to convey an overall feature of the polarization. Moreover, constraints on the production and detection of polarization are expressed very well in terms of the $A_{q}^{(k)}$ basis, and very poorly in terms of $\rho_{M M^{\prime}}$. For detection with $n$ photons, $k$ is limited to $k \leqslant 2 n$; for single photon dissociation,

${ }^{a)}$ Electronic mail: andrew.alexander@ed.ac.uk $|q| \leqslant 2$. When $A_{0}^{(1)}=\sqrt{J /(J+1)}$, the system is said to be maximally oriented along $+z$ : i.e., the $+M_{J}=J$ level is preferentially populated. Likewise, when $A_{0}^{(1)}=-\sqrt{J /(J+1)}$, the system is said to be maximally oriented along $-z$. The $A_{1}^{(1)}$ moment describes orientation perpendicular to $z$. The real part $\operatorname{Re}\left[A_{1}^{(1)}\right]$ describes orientation along $x$, and the imaginary part $\operatorname{Im}\left[A_{1}^{(1)}\right]$ describes orientation along $y$. The polarization parameters are equivalent to spherical tensor moments $\rho_{q}^{(k)}$, and both are easily interconverted, see Appendix A. In this paper we also use $\mathbf{a}_{q}^{(k)}$ to identify a set of polarization parameters in a different frame of reference: the molecule frame, i.e., with respect to the internuclear axis (parallel to the velocity of the photofragment, assuming axial recoil).

Helicity can be defined as the projection of spin angular momentum of a particle along the direction of its linear momentum. We broaden this definition to include total angular momentum J. Orientation implies a distinct helicity, chirality, or handedness, and is therefore a particularly unique probe of the dynamical process (e.g., reactive or dissociative) that generates it. Compared with alignment, relatively few studies have measured fragment orientation. The reason for this, perhaps, is that chiral dynamical processes were expected to be very rare. In practice, however, such processes are being encountered with increasing frequency. For example, orientation can result from photolysis of molecules by circularly polarized light, ${ }^{5-22}$ and by impulsive end-on ("ended") dissociation. ${ }^{23-27}$ It has been found that orientation can be produced by interference between different dissociative channels leading to the same asymptotic fragments, resulting from photodissociation with linearly polarized light. $^{28-36}$ Orientation can also be produced by scattering, from surfaces, ${ }^{37,38}$ or from molecules. ${ }^{39,40}$ The orientation of reaction products has been predicted, but-to our knowledge-not yet observed. ${ }^{41-44}$

The purpose of the present paper is to show how the absolute helicity of a product fragment can be determined, in the context of photodissociation. By absolute helicity, we mean positive or negative, or left and right. Many of the techniques discussed are general, and could be used for studies of scattering or reactions. Beyond the Stern-Gerlach ex- 
periment, most contemporary measurements use circularly (or elliptically) polarized light to determine orientation. Unfortunately, wayward definitions of what is left and right in terms of the polarization of radiation have leaked into studies of molecule dynamics, causing significant confusion. No matter which words have been chosen, parity violation allows us absolutely to distinguish left from right, and to communicate this distinction. ${ }^{45}$ In Sec. II we establish what is meant by left and right circular polarization. Section III gives a physical description of photofragment helicity, resulting from dissociation using either circularly or linearly polarized light. Section IV deals with the production of light of known helicity used in experimental measurements. Section V provides the link between the helicity of circular polarization and fragment helicity, through detected signals. Some concluding remarks are given in Sec. VI.

\section{CIRCULAR POLARIZATION}

We begin by describing what we mean by left and right circular polarization according to the traditional (optics) definitions. $^{46-48}$ The conventional right-handed Cartesian frame is used, with unit vectors $\mathbf{x}, \mathbf{y}, \mathbf{z}$, so that the vector product $\mathbf{x} \times \mathbf{y}=+\mathbf{z}$ holds. The classical plane wave of the light can be written as a function of time $(t), 1,48,49$

$$
\mathbf{E}(t)=\mathbf{e} A \exp \left[i\left(k_{r} z-\omega t\right)\right]
$$

where $A$ is an arbitrary amplitude, e is the polarization vector, $k_{r}=2 \pi / \lambda$ is the wave number ( $\lambda$ is the wavelength), and $\omega=k_{r} v$ is the angular frequency ( $v$ is the wave velocity). In the photon frame the light travels along the $\mathbf{z}$ direction (to $z=+\infty$ ), and the polarization vector lies in the $x y$ plane. For left-circular polarization (LCP),

$$
\mathbf{e}_{\mathrm{LCP}}=(2)^{-1 / 2}(\mathbf{x}+i \mathbf{y})
$$

and for right-circular polarization (RCP),

$$
\mathbf{e}_{\mathrm{RCP}}=-(2)^{-1 / 2}(\mathbf{x}-i \mathbf{y}) .
$$

The definition of LCP is that an observer looking into the oncoming wave would see the electric field $\mathbf{E}$, in a fixed plane $z$, rotate counterclockwise as time increases. ${ }^{46,48}$ This wave is said to have positive helicity $\left(\sigma^{+}\right)$: each photon has a positive projection $(+\hbar)$ along the direction of propagation $(+z) .{ }^{48}$ Conversely, RCP light is said to have negative helicity $\left(\sigma^{-}\right)$: each photon has a negative projection $(-\hbar)$ along the direction of propagation $(+z)$. If we fix $t$, we would see the the distribution of vectors $\mathbf{E}(z)$ trace out a left-handed helix (spiral) in space for LCP, or a right-handed helix for RCP.

Instead of using the Cartesian basis, the polarization vector $\mathbf{e}$ can also be written as a spherical tensor $e_{q}^{(k)}{ }^{1}$ A photon is a spin-1 particle. Generally, a spin-1 particle with welldefined momentum can exist in three possible helicity states $|+1\rangle$, $|0\rangle$, or $|-1\rangle$, corresponding to the projection of spin angular momentum along the direction of the particle's motion. As discussed in Sec. I, a particle with $J=1$ may be described by polarization moments $A_{q}^{(k)}$ where $k=0,1,2$. For light, however, the electric and magnetic fields are transverse to the direction of motion, thereby excluding the $|0\rangle$ helicity state and excluding moments with $k=2$. Also, $e_{0}^{(0)}=1$. There- fore, we can describe the helicity of light by $e_{q}^{(1)}$. The $k=1$ spherical tensor has three components $e_{-1}^{(1)}, e_{0}^{(1)}$, and $e_{+1}^{(1)}$. Following standard definitions (e.g., see Eq. 5.130 of Zare ${ }^{1}$ ), we find that for LCP,

$$
e_{-1}^{(1)}(\mathrm{LCP})=1, \quad e_{+1}^{*(1)}(\mathrm{LCP})=-1,
$$

and for RCP

$$
e_{+1}^{(1)}(\mathrm{RCP})=1, \quad e_{-1}^{*(1)}(\mathrm{RCP})=-1,
$$

with all other components for these tensors identically zero. Note that the $(1, q)$ component of $\mathbf{e}^{*}, e_{q}^{*(1)}=(-1)^{q} e_{-q}^{(1)}$. The fact that the $q=-1$ component of $\mathbf{e}$ is nonzero for LCP (positive helicity) may seem unpalatable: this arises because of the unhappy combination of complex amplitudes and complex basis vectors, and may lead to further confusion. ${ }^{51}$

We can verify that the above definitions produce physically correct results. Consider the matrix element $\langle f|\mathbf{r} \cdot \mathbf{e}| i\rangle$ for an atom absorbing a single photon, within the dipole approximation. ${ }^{52}$ We write $\mathbf{r}$ for the dipole moment operator, and $\mathbf{e}$ for the polarization vector. The photon propagation direction defines the $+\mathbf{z}$ direction of the quantization axis, as above. The initial electronic angular momentum $J$ has projection $M$ on this $z$ axis; the initial state is $|i\rangle=\left|i J_{i} M_{i}\right\rangle$, the final state is $|f\rangle=\left|i J_{f} M_{f}\right\rangle$. In the spherical basis

$$
\mathbf{r} \cdot \mathbf{e}=\sum_{q}(-1)^{q} r_{q}^{(1)} e_{-q}^{(1)}
$$

The matrix element becomes

$$
\begin{aligned}
\langle f|\mathbf{r} \cdot \mathbf{e}| i\rangle= & \sum_{q}(-1)^{q}\left\langle f J_{f} M_{f}\left|r_{q}^{(1)} e_{-q}^{(1)}\right| i J_{i} M_{i}\right\rangle \\
= & (-1)^{J_{f}-M_{f}}\left\langle f J_{f}\left\|r^{(1)}\right\| i J_{i}\right\rangle \\
& \times \sum_{q}(-1)^{q}\left(\begin{array}{ccc}
J_{f} & 1 & J_{i} \\
-M_{f} & q & M_{i}
\end{array}\right) e_{-q}^{(1)},
\end{aligned}
$$

where we have used the Wigner-Eckart theorem for the last step. ${ }^{1}$ The bracketed term $\left(\begin{array}{l}\cdots \\ \cdots\end{array}\right)$ is a $3 J$ symbol: ${ }^{1}$ for it to be nonzero the relation $-M_{f}+q+M_{i}=0$ must hold. For the absorption $\left|i, \frac{1}{2},-\frac{1}{2}\right\rangle \rightarrow\left|f, \frac{1}{2},+\frac{1}{2}\right\rangle$ to occur, we see that $q=+1$ only. The only nonzero term in the sum over $q$ will involve $e_{-1}^{(1)}$, i.e., LCP as above in Eq. (4). This result is consistent with the fact that LCP photons have positive helicity $(+\hbar)$, as required for conservation of the projection of angular momentum along $z$.

\section{PHOTOFRAGMENT ORIENTATION}

The laboratory (lab) frame is chosen to be identical to that of Siebbeles et al., with $\{\theta, \phi\} \equiv\left\{\Theta_{k}, \Phi_{k}\right\}$, see Fig. 1 of Ref. 53. The angles $\{\theta, \phi\}$ are the directions of travel of the photofragments. The major axis $+\mathbf{z}$ of the laboratory frame is defined relative to the photolysis polarization as follows. For photolysis using circular polarization (CP), the light travels in the $+z$ direction of the laboratory frame (towards $z=+\infty$ ). For photolysis using linear polarization (LP), the lab $z$ axis is parallel to the electric field $(\mathbf{E})$ of the light. The molecule frame is defined with the $+\mathbf{z}_{\text {mol }}$ direction as the direction of travel of the fragments; within the axial recoil approxima- 
tion, the $z_{\text {mol }}$ axis is parallel to the internuclear axis. The distribution of the directions of travel of the photofragments in the lab frame can be parameterized by $\beta$, the so-called beta parameter $^{54}$

$$
A_{0}^{(0)}(\theta, \phi)=\frac{1}{4 \pi}\left[1+\beta P_{2}(\cos \theta)\right]
$$

where $P_{2}(\cos \theta)$ is a second Legendre polynomial. Using $\mathrm{CP}$ photolysis, $\beta$ takes limiting values -1 for a pure parallel transition, $+1 / 2$ for a pure perpendicular transition, and intermediate values for some mix of transitions. For LP photolysis these values are multiplied by -2 .

\section{A. Circularly polarized photolysis}

For CP photolysis, the lowest $k=1$ angular momentum orientation moments can be written, ${ }^{53,55,56}$

$$
\begin{aligned}
\rho_{0}^{(1)}(\theta, \phi)= & \frac{3 \sqrt{3}}{4 \pi \sqrt{2 j+1}}\left[a \alpha_{1} \cos ^{2} \theta+a \frac{\gamma_{1}}{2} \sin ^{2} \theta\right], \\
\rho_{1}^{(1)}(\theta, \phi)= & \frac{3 \sqrt{3} e^{i \phi}}{4 \pi \sqrt{2} \sqrt{2 j+1}} \\
& \times \sin \theta \cos \theta\left[-a \alpha_{1}+a \frac{\gamma_{1}}{2}+i \frac{\gamma_{1}^{\prime}}{2}\right],
\end{aligned}
$$

with $\rho_{-1}^{(1)}(\theta, \phi)=-\rho_{1}^{(1)^{*}}(\theta, \phi)$. For LCP light $a=+1$, and for RCP light $a=-1$. Note that the sign of the term in $\gamma_{1}^{\prime}$ does not change sign with the helicity of the photolysis radiation. The above polarization moments are identical to Eqs. (22a) and (22b) of Siebbeles et al. ${ }^{53}$ The polarization moments are equivalent to the complex orientation parameters (see Appendix A),

$$
\begin{aligned}
A_{0}^{(1)}(\theta, \phi)= & \frac{1}{4 \pi}\left[a(1+\beta) \mathbf{a}_{0}^{(1)}(\perp) \cos ^{2} \theta-a \frac{1}{\sqrt{2}}\right. \\
& \left.\times \operatorname{Re}\left[\mathbf{a}_{1}^{(1)}(\|, \perp)\right] \sin ^{2} \theta\right], \\
A_{1}^{(1)}(\theta, \phi)= & -\frac{e^{i \phi}}{8 \pi} \sin \theta \cos \theta\left[a \sqrt{2}(1+\beta) \mathbf{a}_{0}^{(1)}(\perp)\right. \\
& \left.+a \operatorname{Re}\left[\mathbf{a}_{1}^{(1)}(\|, \perp)\right]+i \operatorname{Im}\left[\mathbf{a}_{1}^{(1)}(\|, \perp)\right]\right],
\end{aligned}
$$

with $A_{-1}^{(1)}(\theta, \phi)=-A_{1}^{(1) *}(\theta, \phi)$. In the above equations, we identify various molecule-frame polarization parameters, including the polarization anisotropy parameters $\left\{\alpha_{1}, \gamma_{1}, \gamma^{\prime}{ }_{1}\right\}$ of Vasyutinskii and co-workers, ${ }^{16,55,56}$ and the polarization parameters $\left\{\mathbf{a}_{0}^{(1)}(\perp), \operatorname{Re}\left[\mathbf{a}_{1}^{(1)}(\|, \perp)\right], \operatorname{Im}\left[\mathbf{a}_{1}^{(1)}(\|, \perp)\right]\right\}$ of Rakitzis et al. ${ }^{57}$ The notation $(\|),(\perp)$, and $(\|, \perp)$ are used to distinguish parameters that arise from parallel, perpendicular, and mixed transitions, respectively. However, the dynamical factors $f_{k}\left(q, q^{\prime}\right)$ are those most often obtained in calculations. ${ }^{58}$ By examining Eqs. (9)-(12), we see that $\alpha_{1}$ is similar to $\mathbf{a}_{0}^{(1)}(\perp)$, which describes orientation resulting from perpendicular transitions. Also, $\gamma_{1}$ is similar to $\operatorname{Re}\left[\mathbf{a}_{1}^{(1)}(\|, \perp)\right]$, and $\gamma_{1}^{\prime}$ is similar to $\operatorname{Im}\left[\mathbf{a}_{1}^{(1)}(\|, \perp)\right]$ : these describe orientation from coherent excitation of parallel and perpendicular transitions. Conversion factors between these molecule-frame polarization parameters have been tabulated previously. $^{5}$

As a result of the symmetry of the photolysis polarization vector, the polarization moments in the lab frame should be cylindrically (azimuthally) symmetric around the $z$ axis for both LP and CP photolysis radiations. The presence of $\phi$ in Eqs. (10) and (12) may seem surprising, since we have no definition for $\phi=0$ in the laboratory frame. To visualize the physical distribution of fragment angular momentum in the $\mathrm{lab}$, it is useful to examine the real polarization parameters $A_{q \pm}^{\{k\}}$ using the Hertel-Stoll normalization. ${ }^{59}$ The Hertel-Stoll definitions give $A_{0+}^{\{1\}}=A_{0}^{(1)}, \quad A_{1+}^{\{1\}}$ $=-\sqrt{2} \operatorname{Re}\left[A_{1}^{(1)}\right]$, and $A_{1-}^{\{1\}}=-\sqrt{2} \operatorname{Im}\left[A_{1}^{(1)}\right]$. These real orientation parameters are proportional to the expectation values of the cartesian components of the vector $\mathbf{J},{ }^{60}$ viz.,

$$
A_{1+}^{\{1\}}=\frac{\left\langle J_{x}\right\rangle}{\sqrt{J(J+1)}}, \quad A_{1-}^{\{1\}}=\frac{\left\langle J_{y}\right\rangle}{\sqrt{J(J+1)}}, \quad A_{0+}^{\{1\}}=\frac{\left\langle J_{z}\right\rangle}{\sqrt{J(J+1)}} .
$$

For CP photolysis light, we obtain

$$
\begin{aligned}
\left\langle J_{x}\right\rangle= & C\left[a\left((1+\beta) \mathbf{a}_{0}^{(1)}(\perp)+\frac{1}{\sqrt{2}} \operatorname{Re}\left[\mathbf{a}_{1}^{(1)}(\|, \perp)\right]\right) \cos \phi\right. \\
& \left.-\frac{1}{\sqrt{2}} \operatorname{Im}\left[\mathbf{a}_{1}^{(1)}(\|, \perp)\right] \sin \phi\right] \sin \theta \cos \theta, \\
\left\langle J_{y}\right\rangle= & C\left[a\left((1+\beta) \mathbf{a}_{0}^{(1)}(\perp)+\frac{1}{\sqrt{2}} \operatorname{Re}\left[\mathbf{a}_{1}^{(1)}(\|, \perp)\right]\right) \sin \phi\right. \\
& \left.+\frac{1}{\sqrt{2}} \operatorname{Im}\left[\mathbf{a}_{1}^{(1)}(\|, \perp)\right] \cos \phi\right] \sin \theta \cos \theta,
\end{aligned}
$$

$$
\begin{aligned}
\left\langle J_{z}\right\rangle= & C\left[a(1+\beta) \mathbf{a}_{0}^{(1)}(\perp) \cos ^{2} \theta\right. \\
& \left.-a \frac{1}{\sqrt{2}} \operatorname{Re}\left[\mathbf{a}_{1}^{(1)}(\|, \perp)\right] \sin ^{2} \theta\right],
\end{aligned}
$$

where $C=\sqrt{J(J+1)} / 4 \pi$. We can use these equations to plot a three-dimensional vector field $\left\{\left\langle J_{i}\right\rangle\right\} \equiv\left\{\left\langle J_{x}\right\rangle,\left\langle J_{y}\right\rangle,\left\langle J_{z}\right\rangle\right\}$ of the expectation values along $x, y, z$. For plotting purposes, the terms in $\{\theta, \phi\}$ on the right-hand side of Eqs. (14) can be converted by using the standard conversion from spherical polar to Cartesian coordinates. ${ }^{1}$ The transformation from projections along Cartesian axes to projections in a spherical polar axis system is particularly revealing

$$
\begin{aligned}
& \left\langle J_{r}\right\rangle=a C(1+\beta) \mathbf{a}_{0}^{(1)}(\perp) \cos \theta, \\
& \left\langle J_{\theta}\right\rangle=a C \frac{1}{\sqrt{2}} \operatorname{Re}\left[\mathbf{a}_{1}^{(1)}(\|, \perp)\right] \sin \theta, \\
& \left\langle J_{\phi}\right\rangle=C \frac{1}{\sqrt{2}} \operatorname{Im}\left[\mathbf{a}_{1}^{(1)}(\|, \perp)\right] \sin \theta \cos \theta .
\end{aligned}
$$

The above equations neatly reveal how the lab frame symmetry is related to the molecule-frame parameters $\mathbf{a}_{q}^{(1)}(p)$. Note that the uncertainty principle holds, so we should not 
(a)

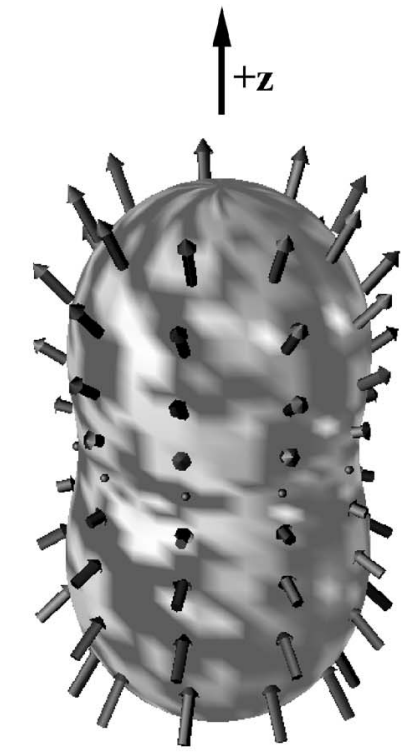

(b)

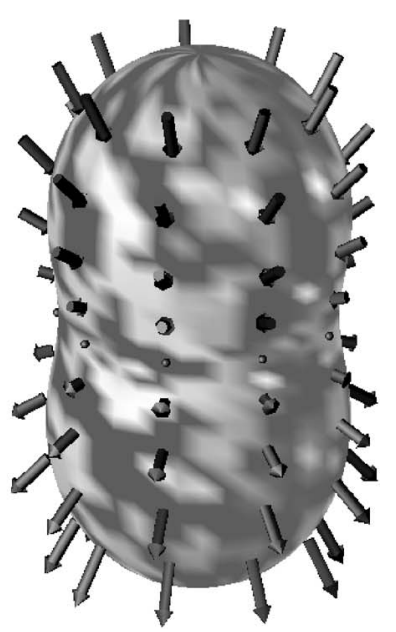

FIG. 1. Plot illustrating the laboratory distribution resulting from the molecule-frame orientation parameter $\mathbf{a}_{0}^{(1)}$, shown as arrows. The laboratory $z$ axis is marked in (a), this is the propagation direction of the circularly polarized photolysis radiation. The arrows represent vectors of expectation values $\left\{\left\langle J_{x}\right\rangle,\left\langle J_{y}\right\rangle,\left\langle J_{z}\right\rangle\right\}$ for total angular momentum $J$. The vector plot is superposed onto an orbital plot of the spatial anisotropy (direction of travel) of the photofragments, Eq. (8), with $\beta=+1 / 2$. If the photolysis radiation is taken as being left circular polarized, then (a) illustrates $\mathbf{a}_{0}^{(1)}>0$, and (b) illustrates $\mathbf{a}_{0}^{(1)}<0$. Alternatively, if we take $\mathbf{a}_{0}^{(1)}>0$, (a) could result from photolysis by left-circular polarized light, and (b) from right-circular polarized light.

take plots of the vector field $\left\{\left\langle J_{i}\right\rangle\right\}$ to be exact representations of the vector field of $\mathbf{J} .^{1,61}$ The vector plots give a better physical picture of the angular momentum polarization of the photofragments, compared with orbital plots: e.g., Fig. 2 of Siebbeles et al. ${ }^{53}$ or Fig. 3 of Torres et al. ${ }^{62}$

Assuming a pure perpendicular transition, the moleculeframe parameters $\operatorname{Re}\left[\mathbf{a}_{1}^{(1)}(\|, \perp)\right]$ and $\operatorname{Im}\left[\mathbf{a}_{1}^{(1)}(\|, \perp)\right]$ are identically zero. Only the molecule frame $\mathbf{a}_{0}^{(1)}(\perp) \neq 0$. We plot the spatial distribution of the vector $\left\{\left\langle J_{i}\right\rangle\right\}$ superimposed onto a plot of the spatial anisotropy (direction of travel) of the products, Eq. (8), represented by a limiting value of the parameter $\beta=+1 / 2$, see Fig. 1. We have taken the photolysis radiation to be LCP, so that $a=+1$ in Eqs. (14). Figure 1(a) shows $\mathbf{a}_{0}^{(1)}(\perp)>0$ and (b) shows $\mathbf{a}_{0}^{(1)}(\perp)<0$. Note that changing helicity of the photolysis light would simply have the effect of reversing the direction of the projections, i.e., reverse the directions of all the arrows in Fig. 1. It is clear that the laboratory distribution is cylindrically symmetric around the $z$ axis (i.e., the propagation direction of the CP photolysis light). Equations (15) reveal that, for this case, the only nonzero projection of angular momentum is the radial projection $\left\langle J_{r}\right\rangle$, as can be seen in Fig. 1.

Some care should be taken in considering angular momentum conservation. For LCP photolysis, the photons carry a projection $+\hbar$ along the direction of propagation. ${ }^{48}$ The helicity of the photolysis radiation must be transferred to the photofragments. However, this does not necessarily fix the sign of $\mathbf{a}_{0}^{(1)}(\perp)$ for every photofragment, because the angular momentum need not be equally shared. In the case of $\mathrm{Cl}_{2}$ photolysis (at ca. $310 \mathrm{~nm}$ ), the orientation parameter $\mathbf{a}_{0}^{(1)}(\perp)$ of the excited-state $\mathrm{Cl}^{*}\left({ }^{2} P_{1 / 2}\right)$ atoms was found to have a sign opposite to the helicity of the photolysis radiation, and opposite to the ground-state $\mathrm{Cl}^{2}\left({ }^{2} P_{3 / 2}\right)$ atoms. ${ }^{18}$

To view the "shape" of the $\operatorname{Re}\left[\mathbf{a}_{1}^{(1)}(\|, \perp)\right]$, we assume that $\mathbf{a}_{0}^{(1)}(\perp)$ and $\operatorname{Im}\left[\mathbf{a}_{1}^{(1)}(\|, \perp)\right]$ are both zero. For an equal mix of parallel and perpendicular transitions excited by $\mathrm{CP}$ photolysis radiation, the spatial anisotropy is represented by $\beta=-1 / 4$. In Fig. 2, we show vector plots superimposed onto an orbital plot representing the spatial anisotropy. Again, we have assumed LCP photolysis radiation $(a=+1)$, so Figs. 2(a) and 2(b) show positive and negative $\operatorname{Re}\left[\mathbf{a}_{1}^{(1)}(\|, \perp)\right]$, respectively. Looking at Eqs. (15), we see that the only nonzero projection for this case is $\left\langle J_{\theta}\right\rangle$, in agreement with Fig. 2.

In the next section, we discuss the physical description of $\operatorname{Im}\left[\mathbf{a}_{1}^{(1)}(\|, \perp)\right]$ in the context of linearly polarized photolysis.

\section{B. Linearly polarized photolysis}

For LP photolysis we write, ${ }^{55,56}$

$$
\rho_{1}^{(1)}(\theta, \phi)=-\frac{3 \sqrt{6} e^{i \phi}}{8 \pi \sqrt{2 j+1}} \sin \theta \cos \theta\left[i \gamma_{1}^{\prime}\right],
$$

and

$$
A_{1}^{(1)}(\theta, \phi)=i \frac{e^{i \phi}}{4 \pi} \sin \theta \cos \theta \operatorname{Im}\left[\mathbf{a}_{1}^{(1)}(\|, \perp)\right],
$$

with $A_{-1}^{(1)}(\theta, \phi)=-A_{1}^{(1) *}(\theta, \phi)$. Note that for LP photolysis, $A_{0}^{(1)}=0$ : for dissociation of an isotropic sample of achiral molecules there can be no overall orientation of $\mathbf{J}$ after integration over all photofragment velocities since the LP light carries no net helicity. As in Sec. III A we calculate expectation values

$$
\begin{aligned}
& \left\langle J_{x}\right\rangle=C \sqrt{2} \operatorname{Im}\left[\mathbf{a}_{1}^{(1)}(\|, \perp)\right] \sin \theta \cos \theta \sin \phi, \\
& \left\langle J_{y}\right\rangle=-C \sqrt{2} \operatorname{Im}\left[\mathbf{a}_{1}^{(1)}(\|, \perp)\right] \sin \theta \cos \theta \cos \phi, \\
& \left\langle J_{z}\right\rangle=0 .
\end{aligned}
$$

Alternatively, in terms of spherical polar projections, 


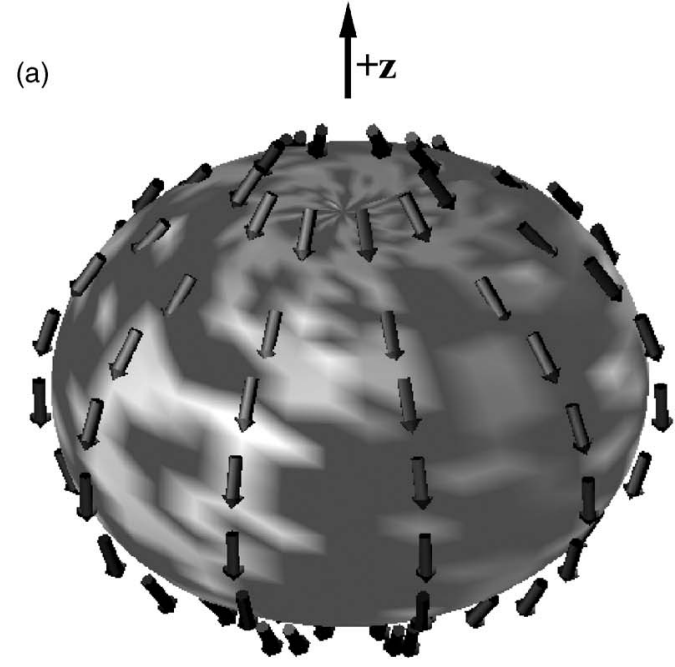

(b)

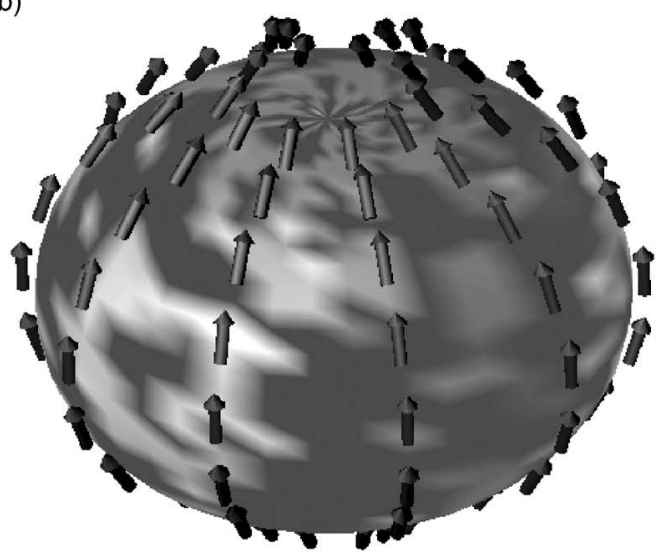

FIG. 2. As for Fig. 1, but illustrating the laboratory distribution resulting from the molecule-frame orientation parameter $\operatorname{Re}\left[\mathbf{a}_{1}^{(1)}(\|, \perp)\right]$. In this plot the spatial anisotropy is characterized by $\beta=-1 / 4$. For left-circular polarized photolysis radiation, (a) shows $\operatorname{Re}\left[\mathbf{a}_{1}^{(1)}(\|, \perp)\right]>0$, and (b) shows $\operatorname{Re}\left[\mathbf{a}_{1}^{(1)}(\|, \perp)\right]<0$.

$$
\left\langle J_{\phi}\right\rangle=-C \sqrt{2} \operatorname{Im}\left[\mathbf{a}_{1}^{(1)}(\|, \perp)\right] \sin \theta \cos \theta,
$$

with $\left\langle J_{r}\right\rangle$ and $\left\langle J_{\theta}\right\rangle$ both identically zero.

For LP photolysis, an equal mix of parallel and perpendicular transitions would correspond to $\beta=+1 / 2$. As above, we plot the vector distributions $\left\{\left\langle J_{i}\right\rangle\right\}$, by superimposing the vector field on a plot of the photofragment spatial anisotropy, see Fig. 3. Now, it can be clearly seen that the projections of $\mathbf{J}$ are distributed with cylindrical symmetry around the LP photolysis electric field $\mathbf{E}$ that defines the $z$ axis, in agreement with Eq. (19). It is also clear that there is no distinction between the two heads of the double-headed photolysis linear polarization vector $\mathbf{E}$ : we may choose either head to define the $+z$ axis. The Schoenflies point group of this distribution is seen to be $D_{\infty}$. To an observer looking from the center of the distribution along the $\mathbf{E}$ vector (towards $z$ $= \pm \infty$ ), the $\mathbf{J}$ are seen to circulate pointing counterclockwise for a positive $\operatorname{Im}\left[\mathbf{a}_{1}^{(1)}(\|, \perp)\right]$ [Fig. 3(a)], and clockwise for a negative $\operatorname{Im}\left[\mathbf{a}_{1}^{(1)}(\|, \perp)\right][$ Fig. 3(b) $]$.

It can be seen that the integrated distribution of $\mathbf{J}$ for a single photofragment has a net helicity of zero. Previously, it was suggested that two partner fragments would be oriented
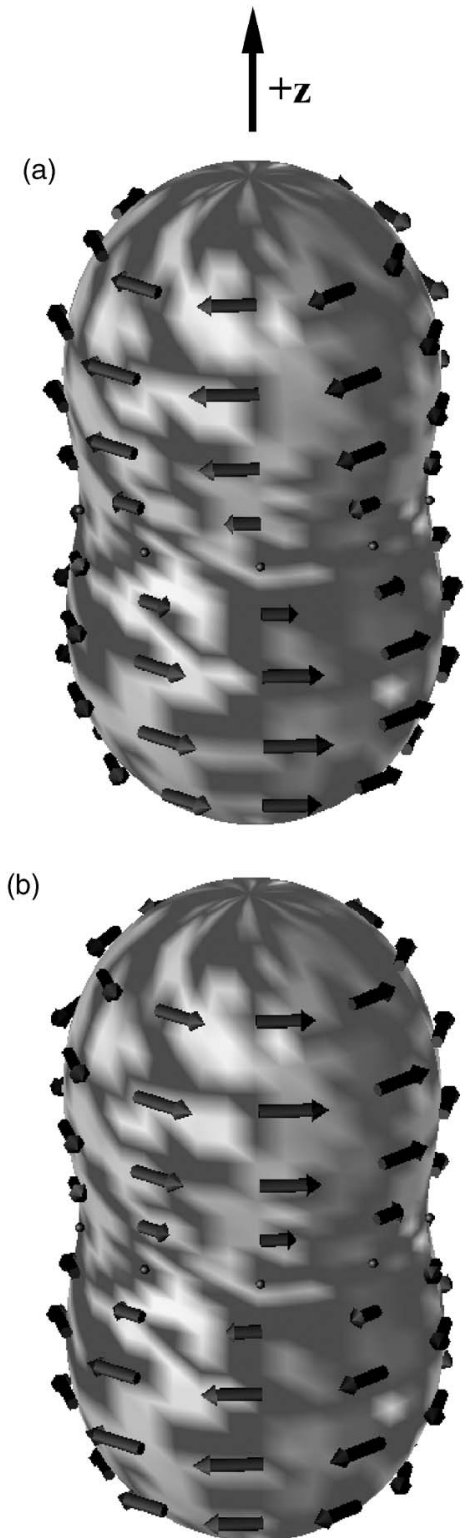

FIG. 3. This figure is similar to Fig. 1, but illustrates the laboratory distribution resulting from the molecule-frame orientation parameter $\operatorname{Im}\left[\mathbf{a}_{1}^{(1)}(\|, \perp)\right]$. The laboratory $z$ axis is marked in (a), this lies along the electric field $\mathbf{E}$ of the linearly polarized photolysis radiation. In this plot the spatial anisotropy is characterized by $\beta=+1 / 2$ (i.e., an equal mix of $\|$ and $\perp$ ). Here (a) shows $\operatorname{Im}\left[\mathbf{a}_{1}^{(1)}(\|, \perp)\right]>0$ [corresponding to, $f_{1}(1,0)<0$ (Refs. 53 and 58)], and (b) shows $\operatorname{Im}\left[\mathbf{a}_{1}^{(1)}(\|, \perp)\right]<0$ [corresponds to $f_{1}(1,0)>0$ ].

exactly opposite to each other. ${ }^{30}$ Indeed, it should be clear that this is not a necessity, and that it is possible for the fragments to have different $\operatorname{Im}\left[\mathbf{a}_{1}^{(1)}(\|, \perp)\right]$. For example, it is possible that a $J=2$ fragment with a nonzero $\operatorname{Im}\left[\mathbf{a}_{1}^{(1)}(\|, \perp)\right]$ is produced along with a cofragment $J=0$ that has $\operatorname{Im}\left[\mathbf{a}_{1}^{(1)}(\|, \perp)\right] \equiv 0$.

\section{EXPERIMENTAL DETERMINATION OF HELICITY}

To measure the absolute helicity of the photofragment orientation parameters, one should know the helicity of the circularly polarized light used to photodissociate the sample. In the following discussion, we assume that the reader is 


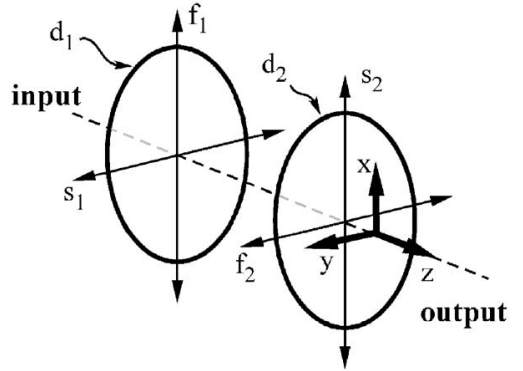

FIG. 4. Schematic figure of a zero-order quarter-wave plate, consisting of two cylindrical slabs of uniaxial crystal with thicknesses $d_{1}$ and $d_{2}$, as marked. Also marked are the double-headed slow $(s)$ and fast $(f)$ axes for both slabs, and the right-handed Cartesian frame $\mathbf{x} \times \mathbf{y}=+\mathbf{z}$. Light is input at the input side, travelling in the $+\mathbf{z}$ direction (i.e., towards $z=+\infty$ ), and exits at the output side. See text for discussion.

familiar with the fundamental principles associated with producing circular polarization, available from optics textbooks: see, e.g., Born and Wolf, ${ }^{46}$ or Fowles. ${ }^{63}$

For commercial quarter-wave $(\lambda / 4)$ retarders, information to determine absolute helicity is not always reliable. On wave plates, some manufacturers mark the fast axis, and others mark the slow: indeed, we know of a manufacturer who has changed their conventions within the last few years. A multiple-order (MO) quarter-wave plate (QWP) is usually made from a single slab of uniaxial crystal (e.g., quartz). Even if we know where are the fast and slow axes of the MO-QWP, the output phase of components polarized along these axes will depend upon the path length through the wave plate. For ease of manufacture and handling, the thickness is usually many multiples of quarter wave, i.e., $\lambda / 4$ $+2 m \lambda$, where integer $m \gg 1$.

In practice, the zero-order ( $\mathrm{ZO}) \mathrm{QWP}$ (with $m=0$ ) is made with two slabs of uniaxial crystal that have different thicknesses, aligned so that the slow axis of one lies along the fast axis of the other. The different thicknesses are chosen to give exactly one quarter wave of retardation at a given wavelength. If the nominal slow (or fast) axis of the combined plate is known, the helicity of circular polarization can be determined. The ZO-QWP is oriented as shown in Fig. 4. The light propagates in the direction $\mathbf{x} \times \mathbf{y}=+\mathbf{z}$. The thickness of the slabs are $d_{1}$ and $d_{2}$. For the uniaxial material, the refractive indices of fast and slow axes are $n_{f}$ and $n_{s}$, respectively (note, $n_{f}<n_{s}$, by definition). The input light is linearly polarized. We take $x$ and $y$ components and find that after passing through the wave plate, the phase of the output components are

$$
\begin{aligned}
& \phi_{x}=\frac{2 \pi}{\lambda}\left(n_{f} d_{1}+n_{s} d_{2}\right), \\
& \phi_{y}=\frac{2 \pi}{\lambda}\left(n_{s} d_{1}+n_{f} d_{2}\right) .
\end{aligned}
$$

Thus, if $d_{1}>d_{2}$ then the $x$ axis will be nominally fast, and $\phi_{x}<\phi_{y}$. The phase difference $\Delta \phi$ is then

$$
\Delta \phi=\phi_{y}-\phi_{x}=\frac{2 \pi}{\lambda}\left(n_{s}-n_{f}\right)\left(d_{1}-d_{2}\right)
$$

To produce output circularly polarized light, the input light should be linearly polarized at $45^{\circ}$ to both the fast and slow axes of the QWP. We write the linearly polarized input light as [cf. Eq. (1)]

$$
\mathbf{E}(t)=(\mathbf{x}+\mathbf{y}) A \exp \left[i\left(k_{r} z-\omega t\right)\right]
$$

The output light will be

$$
\begin{aligned}
& \mathbf{E}^{\prime}(t)=\left(e^{i \phi_{x}} \mathbf{X}+e^{i \phi_{y}} \mathbf{y}\right) A \exp \left[i\left(k_{r} z-\omega t\right)\right] \\
& =e^{i \phi_{x}}\left(\mathbf{x}+e^{i \Delta \phi} \mathbf{y}\right) A \exp \left[i\left(k_{r} z-\omega t\right)\right],
\end{aligned}
$$

where we have assumed that there are no reflection losses. Note that the prefactor $e^{i \phi_{x}}$ is not important, but that the relative phase of the $x$ and $y$ components of polarization is. When $\Delta \phi=\pi / 2$, we have

$$
\mathbf{E}^{\prime}(t)=(\mathbf{x}+i \mathbf{y}) A^{\prime} \exp \left[i\left(k_{r} z-\omega t\right)\right] .
$$

The input polarization $(\mathbf{x}+\mathbf{y})$ has been transformed to output $(\mathbf{x}+i \mathbf{y})$. In a plane at a fixed point $z$, to an observer looking into the approaching light, the electric-field vector will be seen to rotate counterclockwise, as it would for LCP. ${ }^{1,63}$ If the direction of the input linear polarization is changed to $(\mathbf{x}-\mathbf{y})$, we would obtain output RCP.

Using this general treatment, the ZO-QWP can be setup to give light of definite helicity as follows. First, locate the nominal slow or fast axis; ${ }^{64}$ the double-headed slow $(s)$ and fast $(f)$ axes are perpendicular to each other. We choose one head of the fast axis to define the vector $\mathbf{f}$, and one head of the slow to define s. It does not matter which heads are chosen, so long as we have one from each of $f$ and $s$. Then, the ZO-QWP is oriented so the vector product $\mathbf{f} \times \mathbf{s}=\mathbf{z}$, where $+\mathbf{z}$ is the same direction as the propagation vector of the light. Input light linearly polarized along $(\mathbf{f}+\mathbf{s})$ will produce output LCP, and light linearly polarized along $(\mathbf{f}-\mathbf{s})$ will produce RCP.

The cheapest and simplest way of determining the helicity of circular polarization is using a single Fresnel rhomb (FR). The phase changes at each reflection in the rhomb can be calculated from Fresnel's equations. Use of the single FR to determine helicity of light was noted in passing by Kummel et al. $;^{60}$ however, we have been unable to locate a modern reference where the details have been worked out. Although the method is straightforward, for convenience we lay out the details here. We follow the notation used by Fowles, ${ }^{63}$ identical results are obtained by following Born and Wolf. ${ }^{46}$

We place the single Fresnel rhomb in the axis system shown in Fig. 5 The input light propagates in the $+z$ direction, and is translated by the two reflections in the $+x$ direction. We write the electric field exactly as Eq. (21). The input polarization is linear at $45^{\circ}$ so that it will give output circular polarization. With respect to the reflections, light polarized along $y$ would be transverse electric (TE), i.e., $s$ polarized. Light polarized along $x$ would be transverse magnetic (TM), i.e., $p$ polarized. The output light can be written 


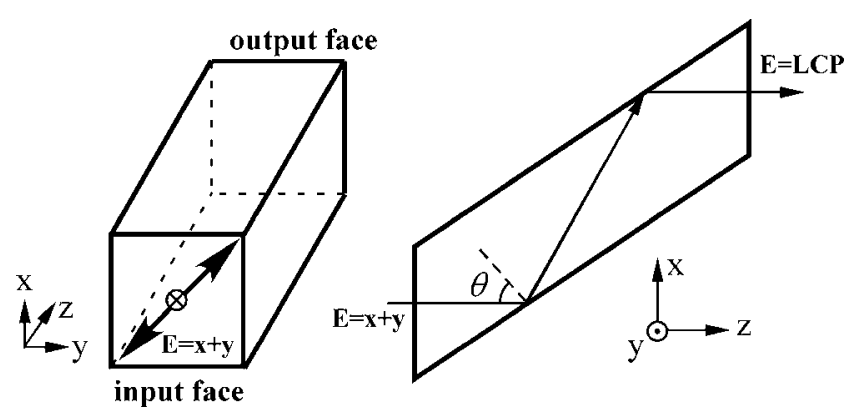

FIG. 5. Schematic figure of the single Fresnel rhomb. See text for detailed discussion. A perspective view is shown on the left, and a side-on view is shown on the right. The right-handed Cartesian frame $\mathbf{x} \times \mathbf{y}=+\mathbf{z}$ is shown. Light is input at the input face, travelling in the $+\mathbf{z}$ direction (i.e., towards $z=+\infty$ ), and exits at the output face. The rhomb is oriented so that light is displaced along the $+\mathbf{x}$ direction, and is not displaced along the $\mathbf{y}$ direction. The angle of incidence at the first reflection $(\theta)$ is shown. The symbol dot-in-circle $(\odot)$ represents a vector pointing toward the reader, out of the page; the symbol cross-in-circle $(\otimes)$ represents a vector pointing away from the reader, into the page.

$$
\mathbf{E}^{\prime}(t)=\left(r_{p}^{2} \mathbf{x}+r_{s}^{2} \mathbf{y}\right) A^{\prime} \exp \left[i\left(k_{r} z-\omega t\right)\right],
$$

where we have twice applied a reflection matrix: $r_{s}$ and $r_{p}$ are the reflection amplitudes. We assume no reflection losses, and ignore the phases obtained by passing through the rhomb. Each internal reflection causes a phase change in the light, the reflection amplitudes can be written $r_{s}=e^{-i \delta_{s}}$ and $r_{p}=e^{-i \delta_{p}}$, where $\delta_{s}$ and $\delta_{p}$ are the phase changes for $s$ - and $p$-polarized lights. ${ }^{63}$ From Eq. (24) we have

$$
\begin{aligned}
\mathbf{E}^{\prime}(t) & =\left(e^{-2 i \delta_{p}} \mathbf{X}+e^{-2 i \delta_{s}}\right) A \exp \left[i\left(k_{r} z-\omega t\right)\right] \\
& =e^{-2 i \delta_{p}}\left(\mathbf{x}+e^{2 i \Delta} \mathbf{y}\right) A \exp \left[i\left(k_{r} z-\omega t\right)\right],
\end{aligned}
$$

where $\Delta=\delta_{p}-\delta_{s}$. Again, note that the relative phase of the $x$ and $y$ polarizations is important. The phase $\Delta$ depends on the geometry of the rhomb, and can calculated to be

$$
\tan \left(\frac{\Delta}{2}\right)=\frac{\cos \theta \sqrt{\sin ^{2} \theta-n^{2}}}{\sin ^{2} \theta},
$$

where $\theta$ is the angle of incidence for the first reflection (Fig. $5)$ and $n=n_{\text {air }} / n_{\text {rhomb }}$ is the ratio of refractive indices. The geometry and material of the FR are chosen so that $\Delta$ $=\pi / 4$, and $e^{2 i \Delta}=+i$. Finally, the output light can be written

$$
\mathbf{E}^{\prime}(t)=(\mathbf{x}+i \mathbf{y}) A^{\prime} \exp [i(k z-\omega t)]
$$

The input polarization $(\mathbf{x}+\mathbf{y})$ has been transformed to output $(\mathbf{x}+i \mathbf{y})$, i.e., LCP light. ${ }^{1,63}$ If the direction of the input linear polarization is changed to $(\mathbf{x}-\mathbf{y})$, we would obtain output right-circular polarization.

The FR can be used to calibrate other quarter-wave plates as follows. If, instead of linearly polarized light, we input RCP light $(\mathbf{x}-i \mathbf{y})$ into the FR oriented as above, we would obtain output linearly polarized light $(\mathbf{x}+\mathbf{y})$. Alternatively, input LCP would produce output $(\mathbf{x}-\mathbf{y})$. The output linear polarizations $(\mathbf{x} \pm \mathbf{y})$ are readily distinguished in the laboratory frame using a linear polarizer whose polarization axis is known. By inputing $\mathrm{CP}$ light of indeterminate sign into the FR, we can determine whether the input light is left or right by measuring which output linear polarization is obtained.
TABLE I. Values of the contracted polarization tensor $E_{q}^{(k)}$ in the photon frame for linearly and circularly polarized lights (Ref. 1). Recall that in the photon frame, the $+\mathbf{z}$ direction is the propagation direction of the light for circular polarization (left or right), and $\mathbf{z}$ lies along the electric field $\mathbf{E}$ for linear polarization.

\begin{tabular}{lccc}
\hline \hline$E_{q}^{(k)}$ & LP & LCP & RCP \\
\hline$E_{0}^{(0)}$ & $-\frac{1}{\sqrt{3}}$ & $-\frac{1}{\sqrt{3}}$ & $-\frac{1}{\sqrt{3}}$ \\
$E_{0}^{(1)}$ & 0 & $\frac{1}{\sqrt{2}}$ & $-\frac{1}{\sqrt{2}}$ \\
$E_{0}^{(2)}$ & $\frac{2}{\sqrt{6}}$ & $-\frac{1}{\sqrt{6}}$ & $-\frac{1}{\sqrt{6}}$
\end{tabular}

\section{CALCULATION OF SENSITIVITY OF POLARIZED DETECTION SCHEMES}

Contemporary photofragment polarization measurements are normally made by detection schemes using polarized light. There are a number of polarized detection schemes in use, particularly resonantly enhanced multiphoton ionization (REMPI) and laser-induced fluorescence (LIF), although other schemes such as degenerate four-wave mixing are possible. ${ }^{65}$ In general, the signal intensity $I$ can be written ${ }^{60,66}$

$$
I=C \sum_{k, q} P_{q}^{(k)}\left(J_{i}, J_{f} ; \Omega_{\mathrm{lab}}\right) A_{q}^{(k)},
$$

where $C$ is a common factor, $J_{i}$ is the ground-level, the level $J_{f}$ is the final resonant state, and $\Omega_{\text {lab }}$ represents a set of factors that describe the geometry of the detection scheme, including the polarization of light used. In this equation, the frame is defined by the parameters $A_{q}^{(k)}$ : these may have been produced by a chemical reaction, or as the result of a photodissociation. The sensitivity moments $P_{q}^{(k)}\left(J_{i}, J_{f} ; \Omega_{\text {lab }}\right)$ can be calculated for a given experimental detection scheme, and allow the extraction of the polarization parameters $A_{q}^{(k)}$ from measured intensities.

To calculate $P_{q}^{(k)}\left(J_{i}, J_{f} ; \Omega_{\mathrm{lab}}\right)$ for the orientation moments (odd $k$ ), some care should be taken in describing the circular (or elliptical) polarization of the light used. For many detection schemes, it is convenient to use the contracted polarization tensor $E_{q}^{(k)}$ to calculate the sensitivity moments, ${ }^{1,60,67}$

$$
\begin{aligned}
E_{q}^{k} & =\left[\mathbf{e} \otimes \mathbf{e}^{*}\right]_{q}^{(k)} \\
& =(-1)^{q} \sqrt{2 k+1} \sum_{m} e_{m}^{(1)} e_{q-m}^{*(1)}\left(\begin{array}{ccc}
1 & 1 & k \\
m & q-m & -q
\end{array}\right) .
\end{aligned}
$$

We caution the reader to note that $\left[\mathbf{e}^{*} \otimes \mathbf{e}\right]_{q}^{(k)}=(-1)^{k}[\mathbf{e}$ $\left.\otimes \mathbf{e}^{*}\right]_{q}^{(k)}$ : the former is commonly used for absorption, the latter for emission. In Table I, we have tabulated $E_{q}^{(k)}$ in the photon frame (Sec. II) for CP and LP lights.

For polarized two-photon REMPI, it is convenient to use the much-simplified sensitivity factor of Mo and Suzuki, in the probe laser photon frame, ${ }^{68}$ 


$$
\begin{aligned}
\widetilde{P}_{k}= & 5 \sqrt{(2 k+1)\left(2 J_{i}+1\right)}(-1)^{J_{i}+J_{f}}\left(\begin{array}{ccc}
2 & 2 & k \\
2 s & -2 s & 0
\end{array}\right) \\
& \times\left\{\begin{array}{ccc}
J_{i} & J_{i} & k \\
2 & 2 & J_{f}
\end{array}\right\} .
\end{aligned}
$$

This expression can be used for any valid $J_{i} \rightarrow J_{f}$ transition using CP light. The bracketed term $\{\cdots\}$ is a $6 J$ symbol. ${ }^{1}$ It may be that the frame of the parameters $A_{q}^{(k)}$ that we wish to measure does not coincide with the photon frame, in which case a transformation should be made (cf. Eq. (2) of Ref. 68). The intensity will then be given by

$$
I=C \sum_{k} s_{k} A_{0}^{(k)}(\mathrm{pr})=C \sum_{k, q} s_{k} A_{q}^{(k)}(\mathrm{lab}) C_{k q}^{*}(\Theta, \Phi),
$$

where $A_{q}^{(k)}(\mathrm{lab})$ are lab frame parameters, and $A_{0}^{(k)}(\mathrm{pr})$ are the parameters in the corresponding probe photon frame. The sensitivity factor $s_{k}$ can be obtained by conversion of $\widetilde{P}_{k}$ of Eq. (30) above, according to Eq. (6) of Ref. 36. $C_{k q}^{*}(\Theta, \Phi)$ is a complex conjugate of a modified spherical harmonic, ${ }^{1}$ and $\{\Theta, \Phi\}$ are polar angles in the lab frame that describe the $z_{\mathrm{pr}}$ axis of the probing light. The $z_{\mathrm{pr}}$ axis is given by the propagation direction for $\mathrm{CP}$ light, or the direction of the $\mathbf{E}$ field for LP light.

For Eq. (30) we determine that $s=+1$ for LCP and $s=-1$ for RCP. The correct signs for $s$ can be verified by replacing the spherical tensor components $e_{q}^{(1)}$ for LCP and RCP [Eqs. (4) and (5) above] into Eq. (4) of Mo and Suzuki. ${ }^{68}$ Alternatively, the sign of $s$ can be determined by analysis of the relevant $3 J$ symbols in their derivation: in Eq. (8) of Ref. 68 , we find $3 J$ symbols like

$$
\left(\begin{array}{ccc}
J_{i} & J_{f} & T \\
M_{i} & -M_{f} & 2 s
\end{array}\right) .
$$

Here $M_{i}, M_{f}$ are the magnetic sublevels for $J_{i}$ and $J_{f}$. It is found that $T=2$ for CP light. ${ }^{68}$ We choose $J_{i}=3 / 2, J_{f}=1 / 2$, and $M_{i}=+3 / 2$ so that all of the population initially is in the $+3 / 2$ magnetic sublevel (i.e., fully oriented). From the $3 J$ symbol we see the selection rule $M_{f}=M_{i}+2 s$; thus, $M_{f}=-1 / 2$ if $s=-1$, and $M_{f}=+7 / 2$ if $s=+1$. There can be no $M_{f}=+7 / 2$ sublevel for $J_{f}=1 / 2$; thus, $s=-1$. But what polarization can drive the $M_{i}=+3 / 2 \rightarrow M_{f}=-1 / 2$ absorption? The particle must absorb the projection of $-2 \hbar$ of angular momentum: so, we determine that $s=-1$ corresponds to RCP. This method is probably the safest way to test similar intensity expressions where the sign of helicity is not explicitly connected to LCP or RCP (see, e.g., Refs. 69 and 70).

\section{CONCLUDING REMARKS}

In the present paper we have shown how the absolute helicity (positive or negative) of oriented photofragments can be measured. The symmetry of distributions of oriented photofragments has been visualized using three-dimensional vector plots of expectation values of the total angular momenta of the fragments. The vector plot neatly illustrates the symmetry of the laboratory frame distributions of photofragment angular momenta in terms of the molecule-frame orientation parameters. The description of circular polarization in the spherical tensor basis has been discussed. Procedures for the determination of the absolute helicity (positive or negative, left or right) of circularly polarized light produced by quarter-wave plates and a single Fresnel rhomb have been given, and the use of circular polarization for optical measurement of the orientation of fragments has been discussed. It is hoped that the present work will help to clarify some of the confusion that has arisen over what is left and right in terms of orientation and circular polarization. We hope that the methods discussed here will facilitate future experimental measurements of fragment helicity, in photodissociation, scattering, and reactive systems.

\section{ACKNOWLEDGMENTS}

The author thanks David Van Baak (Calvin College), Alex Brown (University of Alberta), Dmitry Budker (UC, Berkeley), Matt Costen (Heriot-Watt University), Marcelo de Miranda (University of Leeds), Arthur Suits (Wayne State University), and Oleg Vasyutinskii (Ioffe Institute, St. Petersburg) for varied and useful discussions. The author also thanks The Royal Society for financial support and a University Research Fellowship.

\section{APPENDIX: CONVERSION FACTORS}

The angular momentum polarization moments $\rho_{q}^{(k)}(\theta, \phi)$ are equivalent to the $T_{K Q}\left(\Theta_{k}, \Phi_{k}\right)$ of Ref. 53, and can be converted to the complex polarization parameters $A_{q}^{(k)}(\theta, \phi)$ using the following expression:

$$
A_{q}^{(k)}(\theta, \phi)=\frac{c(k)\left\langle J|| J^{(k)}|| J\right\rangle}{[J(J+1)]^{k / 2} \sqrt{2 k+1}} \rho_{q}^{(k)}(\theta, \phi) .
$$

General expressions for the conversion factors $c(k)$, and the reduced matrix elements $\left\langle J\left\|J^{(k)}\right\| J\right\rangle$ are given by van Vroonhoven and Groenenboom. ${ }^{71}$ Note that the $\rho_{q}^{(k)}$ are not state multipole moments in the conventional sense, ${ }^{1,4}$ since they have been obtained by covariant expansion over state multipoles. The polarization moments $\rho_{q}^{(k)}(\theta, \phi)$ used here can be converted to the state multipole moments $\widetilde{\rho}_{q}^{(k)}(\theta, \phi)$ with the relation $\rho_{q}^{(k)}(\theta, \phi)=(-1)^{q} \widetilde{\rho}_{-q}^{(k)}(\theta, \phi)$.

${ }^{1}$ R. N. Zare, Angular Momentum: Understanding Spatial Aspects in Chemistry and Physics (Wiley, New York, 1988).

${ }^{2}$ A. J. Alexander and R. N. Zare, Acc. Chem. Res. 33, 199 (2000).

${ }^{3}$ A. J. Orr-Ewing and R. N. Zare, Annu. Rev. Phys. Chem. 45, 315 (1994).

${ }^{4}$ K. Blum, Density Matrix Theory and Applications, 2nd ed. (Plenum, New York, 1996).

${ }^{5}$ A. J. Alexander, J. Chem. Phys. 118, 6234 (2003).

${ }^{6}$ O. S. Vasyutinskii, JETP Lett. 31, 456 (1980).

${ }^{7}$ O. S. Vasyutinskii, Sov. Phys. JETP 54, 855 (1981).

${ }^{8}$ H. Katô and K. Onomichi, J. Chem. Phys. 82, 1642 (1985).

${ }^{9}$ E. Hasselbrink, J. R. Waldeck, and R. N. Zare, Chem. Phys. 126, 191 (1988).

${ }^{10}$ J. Vigué, B. Girard, G. Gouédard, and N. Billy, Phys. Rev. Lett. 62, 1358 (1989).

${ }^{11}$ J. F. Black, E. Hasselbrink, J. R. Waldeck, and R. N. Zare, Mol. Phys. 71, 1143 (1990).

${ }^{12}$ D. V. Kupriyanov, B. N. Sevastianov, and O. S. Vasyutinskii, Z. Phys. D: At., Mol. Clusters 15, 105 (1990).

${ }^{13}$ J. A. Beswick, M. Glass-Maujean, and O. Roncero, J. Chem. Phys. 96, 7514 (1992).

${ }^{14}$ D. V. Kupriyanov, B. V. Picheyev, and O. S. Vasyutinskii, J. Phys. B 26, L803 (1993). 
${ }^{15}$ A. G. Evseev, D. V. Kupriyanov, B. V. Picheyev, B. N. Sevastianov, and O. S. Vasyutinskii, Chem. Phys. 171, L803 (1993).

${ }^{16}$ B. V. Picheyev, A. G. Smolin, and O. S. Vasyutinskii, J. Phys. Chem. A 101, 7614 (1997).

${ }^{17}$ M. L. Costen, Faraday Discuss. 113, 455 (1999).

${ }^{18}$ A. J. Alexander, Z. H. Kim, S. A. Kandel, R. N. Zare, T. P. Rakitzis, Y Asano, and S. Yabushita, J. Chem. Phys. 113, 9022 (2000).

${ }^{19}$ A. J. Alexander, Phys. Rev. A 66, 060702 (2002).

${ }^{20}$ T. P. Rakitzis, P. C. Samartzis, R. L. Toomes, T. N. Kitsopoulos, A. Brown, G. G. Balint-Kurti, O. S. Vasyutinskii, and J. A. Beswick, Science 300, 1936 (2003).

${ }^{21}$ T. P. Rakitzis, P. C. Samartzis, R. L. Toomes, and T. N. Kitsopoulos, J. Chem. Phys. 121, 7222 (2004).

${ }^{22}$ S. K. Lee, D. Townsend, O. S. Vasyutinskii, and A. G. Suits, Phys. Chem. Chem. Phys. 7, 1650 (2005)

${ }^{23}$ V. K. Nestorov and J. I. Cline, J. Chem. Phys. 111, 5287 (1999).

${ }^{24}$ M. Brouard, P. O'Keefe, D. M. Joseph, and D. Minayev, Phys. Rev. Lett. 86, 2249 (2001)

${ }^{25}$ G. Hancock, P. J. Pearson, G. A. D. Ritchie, and D. F. Tibbets, Phys. Chem. Chem. Phys. 5, 5386 (2003).

${ }^{26}$ G. Hancock, P. J. Pearson, G. A. D. Ritchie, and D. F. Tibbets, Chem. Phys. Lett. 393, 425 (2004).

${ }^{27}$ G. Hancock, S. J. Horrocks, P. J. Pearson, G. A. D. Ritchie, and D. F. Tibbets, J. Chem. Phys. 122, 244321 (2005).

${ }^{28}$ L. D. A. Siebbeles and J. A. Beswick, J. Chem. Soc., Faraday Trans. 88 2565 (1992).

${ }^{29}$ O. S. Vasyutinskii, A. G. Evseev, D. V. Kupriyanov, B. N. Sevastyanov, and B. V. Picheyev, Zh. Eksp. Teor. Fiz. 103, 758 (1993).

${ }^{30}$ T. P. Rakitzis, S. A. Kandel, A. J. Alexander, Z. H. Kim, and R. N. Zare, Science 281, 1346 (1998).

${ }^{31}$ T. P. Rakitzis, S. A. Kandel, A. J. Alexander, Z. H. Kim, and R. N. Zare, J. Chem. Phys. 110, 3351 (1999).

${ }^{32}$ Z. H. Kim, A. J. Alexander, S. A. Kandel, T. P. Rakitzis, and R. N. Zare, Faraday Discuss. 113, 27 (1999).

${ }^{33}$ Z. H. Kim, A. J. Alexander, and R. N. Zare, J. Phys. Chem. A 103, 10144 (1999)

${ }^{34}$ E. R. Wouters, M. Beckert, L. J. Russell, K. N. Rosser, A. J. Orr-Ewing, M. N. R. Ashfold, and O. S. Vasyutinskii, J. Chem. Phys. 117, 2087 (2002).

${ }^{35}$ Y. Kimura, S. Kasahara, H. Kato, and M. Baba, Phys. Rev. A 67, 062717 (2003).

${ }^{36}$ A. J. Alexander, Z. H. Kim, and R. N. Zare, J. Chem. Phys. 118, 10566 (2003).

${ }^{37}$ G. O. Sitz, A. C. Kummel, and R. N. Zare, J. Chem. Phys. 87, 3247 (1987).

${ }^{38}$ G. O. Sitz, A. C. Kummel, R. N. Zare, and J. C. Tully, J. Chem. Phys. 89, 2572 (1988)

${ }^{39}$ C. P. Fell, A. J. McCaffery, K. L. Reid, and A. Ticktin, J. Chem. Phys. 95, 4948 (1991)

${ }^{40}$ K. T. Lorenz, D. W. Chandler, J. W. Barr, W. W. Chen, G. L. Banares, and J. I. Cline, Science 293, 2063 (2001).

${ }^{41}$ F. J. Aoiz, M. Brouard, V. J. Herrero, V. Sáez Rabános, and K. Stark, Chem. Phys. Lett. 264, 487 (1997)

${ }^{42}$ T. P. Rakitzis, S. A. Kandel, T. Lev-On, and R. N. Zare, J. Chem. Phys. 107, 9392 (1997).

${ }^{43}$ A. J. Alexander, F. J. Aoiz, L. Bañares, M. Brouard, J. Short, and J. P. Simons, J. Phys. Chem. A 101, 7544 (1997).

${ }^{44}$ M. P. de Miranda, F. J. Aoiz, L. Bañares, and V. Sáez Rabános, J. Chem. Phys. 111, 5368 (1999).

${ }^{45}$ M. Gardner, The New Ambidextrous Universe: Symmetry and Asymmetry, from Mirror Reflections to Superstrings, 3rd ed. (W. H. Freeman \& co., New York, 1991).

${ }^{46} \mathrm{M}$. Born and E. Wolf, Principles of Optics, 7th ed. (Cambridge University Press, Cambridge, 2002)

${ }^{47}$ E. Hecht, Optics, 4th ed. (Addison-Wesley, San Francisco, 2002).

${ }^{48}$ J. D. Jackson, Classical Electrodynamics, 3rd ed. (Wiley, New York, 1998).
${ }^{49}$ We give a description in terms of classical electrodynamics. In Eq. (1) we have written the positive frequency component of the free field; the negative frequency component of the free field is given by the complex conjugate of Eq. (1). In terms of quantum electrodynamics, e appears beside the photon annihilation operator $a$ (i.e., for absorption), and $\mathbf{e}^{*}$ beside the photon creation operator $a^{\dagger}$ (i.e., for emission) (Ref. 50).

${ }^{50}$ C. Cohen-Tannoudji, J. Dupont-Roc, and G. Grynberg, Photons and Atoms: Introduction to Quantum Electrodynamics (Wiley, New York, 1997).

${ }^{51}$ We offer the following errata to Zare, p. 209 (Ref. 1). The right-hand side of Eq. 5.118 should read $-(2)^{-1 / 2}(\hat{\mathbf{X}}-i \hat{\mathbf{Y}})$. In the text that follows change " $-e(1,1)$ " to " $e(1,-1)=1$ " ( $\mathrm{LCP})$ and " $e(1,-1)$ " to " $e(1,1)=1$ " (RCP). The next paragraph should read "...e(1,1) $=-(\cos \beta-\sin \beta) / \sqrt{2}, e(1$, $-1)=(\cos \beta+\sin \beta) / \sqrt{2}, \quad e^{*}(1,1)=-(\cos \beta+\sin \beta) / \sqrt{2}, \quad$ and $\quad e^{*}(1,-1)$ $=(\cos \beta-\sin \beta) / \sqrt{2}$."

${ }^{52}$ D. Budker, D. F. Kimball, and D. P. DeMille, Atomic Physics: an exploration through problems and solutions (Oxford University Press, Oxford, 2004).

${ }^{53}$ L. D. A. Siebbeles, O. S. Glass-Maujean, O. S. Vasyutinskii, J. A. Beswick, and O. Roncero, J. Chem. Phys. 100, 3610 (1994).

${ }^{54}$ R. N. Zare and D. R. Herschbach, Proc. IEEE 51, 173 (1963).

${ }^{55}$ A. S. Bracker, E. R. Wouters, A. G. Suits, and O. S. Vasyutinskii, J. Chem. Phys. 110, 6749 (1999).

${ }^{56}$ E. R. Wouters, M. Ahmed, D. S. Peterka, A. S. Bracker, A. G. Suits, and O. S. Vasyutinskii, in Imaging in Chemical Dynamics, edited by A. G. Suits and R. E. Continetti (American Chemical Society, Washington, DC, 2000).

${ }^{57}$ T. P. Rakitzis and R. N. Zare, J. Chem. Phys. 110, 3341 (1999). Note, (II), $(\perp)$, and $(\|, \perp)$ are used to distinguish between parallel, perpendicular, and mixed transitions, respectively.

${ }^{58}$ G. G. Balint-Kurti, A. J. Orr-Ewing, J. A. Beswick, A. Brown, and O. S. Vasyutinskii, J. Chem. Phys. 116, 10760 (2002).

${ }^{59}$ I. V. Hertell and W. Stoll, Adv. At. Mol. Phys. 13, 113 (1978). The Hertel-Stoll definitions are $A_{q+}^{\{k\}}=1 / \sqrt{2}\left[(-1)^{q} A_{q}^{(k)}+A_{-q^{(k)}}^{(k)}\right.$ and $A_{q-}^{\{k\}}=(1 / i \sqrt{2})$ $\times\left[(-1)^{q} A_{q}^{(k)}-A_{-q}^{(k)}\right]$, for $q>0$. Also, $A_{0+}^{\{k\}}=A_{0}^{(k)}$ and $A_{0-}^{\overline{\{k}\}}=0$, for $q=0$.

${ }^{60}$ A. C. Kummel, G. O. Sitz, and R. N. Zare, J. Chem. Phys. 88, 6707 (1988). See Table VI.

${ }^{61}$ M. P. de Miranda, F. J. Aoiz, V. Sáez-Rábanos, and M. Brouard, J. Chem. Phys. 121, 9830 (2004).

${ }^{62}$ E. A. Torres, D. A. Baugh, and R. D. Levine, Chem. Phys. Lett. 375, 141 (2003).

${ }^{63}$ G. R. Fowles, Introduction to Modern Optics, 2nd ed. (Dover, New York, 1989). Note that the reflection coefficient $r_{p}$ has opposite sign to Born and Wolf.

${ }^{64}$ The following procedure may be used to distinguish between fast $(f)$ and slow $(s)$ axes for a quarter-wave plate (QWP). Find the $f / s$ axes by placing the QWP between two crossed vertical and horizontal linear polarizers. Looking through the optics at a white light source, rotate the QWP to get maximum transmission: the $f$ and $s$ axes will be at $\pm 45^{\circ}$ from the horizontal. Now tilt the QWP about one of these ( $f$ or $s$ ) axes, so that the light passes through a thicker path of the QWP. If the color of light changes through grey to black, then the QWP is being tilted about the $f$ axis. If the color changes from white to multiple (interference) colors, then the QWP is being tilted about the $s$ axis. Note that this test may not work for longer-wavelength (infrared) QWPs.

${ }^{65}$ T. A. W. Wasserman, P. H. Vaccaro, and B. R. Johnson, J. Chem. Phys. 108, 7713 (1998).

${ }^{66}$ D. A. Case, G. M. McClelland, and D. R. Herschbach, Mol. Phys. 35, 541 (1978).

${ }^{67}$ A. C. Kummel, G. O. Sitz, and R. N. Zare, J. Chem. Phys. 88, 7357 (1988). Erratum: in Table III, $E_{0}^{1}$ for case A should read " $(-1 / \sqrt{2}) \sin 2 \beta$."

${ }^{68}$ Y. Mo and T. Suzuki, J. Chem. Phys. 109, 4691 (1998).

${ }^{69}$ A. J. Bain and A. J. McCaffery, J. Chem. Phys. 80, 5883 (1984).

${ }^{70}$ A. J. Bain and A. J. McCaffery, J. Chem. Phys. 83, 2627 (1985); J. Chem. Phys. 83, 2732 (1985); J. Chem. Phys. 83, 2641 (1985).

${ }^{71}$ M. C. G. N. van Vroonhoven and G. C. Groenenboom, J. Chem. Phys. 116, 1965 (2002). 